\title{
Evaluation of left ventricular diastolic function by fractional area change using cine cardiovascular magnetic resonance: a feasibility study
}

\author{
Satoshi Okayama ${ }^{1 *}$, Tomoya Nakano $^{1 \dagger}$, Shiro Uemura ${ }^{1 \dagger}$, Shinichi Fujimoto ${ }^{2 \dagger}$, Satoshi Somekawa ${ }^{1 \dagger}$,
} Makoto Watanabe ${ }^{3 \dagger}$, Tamio Nakajima ${ }^{3+}$ and Yoshihiko Saito ${ }^{1+}$

\begin{abstract}
Background: Evaluation of left ventricular (LV) diastolic function is essential for the management of heart failure. We verified whether LV diastolic function could be evaluated by measuring the fractional area change (FAC) using cine cardiovascular magnetic resonance (CMR).

Methods: We collected clinical data from 59 patients who underwent echocardiography and cine CMR. Normal, impaired relaxation, pseudonormal, and restrictive LV filling were observed in $15,28,11$, and 5 patients, respectively. We calculated FAC during the first 30\% of diastole (diastolic-index\%) in the short-axis view, by tracing the contours on only three MR cine images.

Results: The diastolic index was significantly lower $(p<0.0001)$ in patients with impaired relaxation $(32.4 \pm 7.5)$, pseudonormal filling (25.4 \pm 5.6$)$, and restrictive filling $(9.5 \pm 1.5)$ compared to those with normal diastolic function $(67.7 \pm 10.8)$, and the index decreased significantly with worsening of diastolic dysfunction. The diastolic index correlated positively with early diastolic mitral annular velocity measured by tissue Doppler imaging $(r=0.75$, $p<0.0001$ ), respectively.

Conclusions: Measurement of FAC can be useful for the evaluation of LV diastolic function using cine CMR. Keywords: Cine, Diastolic function, Relaxation, Pseudonormal, Echocardiography, Color kinesis
\end{abstract}

\section{Background}

The accurate evaluation of left ventricular (LV) diastolic as well as systolic function is essential for the diagnosis and treatment of heart failure. Impaired LV diastolic function often precedes systolic dysfunction in heart failure and is observed at its earliest stage [1]. It is associated with increased end-diastolic pressure, dyspnoea, fatigue, and reduced exercise tolerance [2] and is closely associated with increased mortality [3]. Currently, echocardiography is the main noninvasive modality used for the evaluation of LV diastolic function [4].

On the other hand, cardiovascular magnetic resonance (CMR) has been widely used for the evaluation of left ventricular (LV) morphology and function due to its

\footnotetext{
* Correspondence: satosi01@naramed-u.ac.jp

${ }^{\dagger}$ Equal contributors

${ }^{1}$ First Department of Internal Medicine, Nara Medical University, Nara, Japan Full list of author information is available at the end of the article
}

excellent image quality and lack of geometric assumptions. However, this modality is less popular for evaluating diastolic function despite the development of several relevant techniques, including the use of volumetric filling curves [5], phase-contrast imaging [6], tagging [7], and strain-encoded imaging [8]. This might be because these acquisition sequences or software for automatically measuring LV diastolic function are not yet applied generally $[5,9]$.

Regional wall motion has also been evaluated using a color kinesis echocardiography system (Philips Medical Systems, Andover, MA, USA) [10]. This method can track and display endocardial motion by automatically tracing endocardial contours and evaluate LV diastolic function by measuring the degree of expansion during the first $30 \%$ of diastole in the short-axis view [11-15].

The purposes of this study were to apply the concepts of echocardiographic color kinesis (Echo-CK) to cine CMR 
and to determine whether measurement of LV fractional area change can enable easy evaluation of diastolic as well as systolic functions. Accordingly, cardiac function indices obtained from the measurement of LV fractional area change in cine CMR were compared to conventional echocardiographic measurements.

\section{Methods}

\section{Subjects}

We collected clinical data from consecutive patients who underwent cardiac MRI and transthoracic echocardiography for evaluation of LV diastolic function between January 1, 2010 and December 31, 2012 at Hirai Hospital. The exclusion criteria were as follows: (1) unfit for accurate evaluation by echocardiography, (2) arrhythmia, (3) difficulty in breath-holding, (4) pericardial disease, (5) moderate-to-severe aortic or mitral regurgitation assessed with color-flow Doppler echocardiography, and (6) mitral valve stenosis. Thus, we retrospectively evaluated 59 patients (41 men and 18 women, mean age $59.5 \pm 18.9$ years) with excellent-quality MR cine images. The present study conformed to the clinical research protocols approved by the institutional ethics committee of Hirai Hospital, in compliance with the 1975 Declaration of Helsinki.

\section{Echocardiography}

LV systolic function was assessed by calculating ejection fraction (EF) with the Modified-Simpson method. LV and left atrial (LA) dimensions were measured by using M-mode echocardiography.

Next, diastolic function was assessed by transmitral and tissue Doppler imaging at the septal mitral annulus. Peak early (E) and late transmitral filling velocities (A), their ratio, the deceleration time (DCT) of peak E velocity, early diastolic mitral annular velocity $(\mathrm{Ea})$, and $\mathrm{E} / \mathrm{Ea}$ were measured. The subjects were classified into the following four groups according to the Recommendations for the Evaluation of Left Ventricular Diastolic Function by the American Society of Echocardiography [16]: (1) a normal diastolic function group with normal ejection fraction $(\geq 60 \%)$ and increased Ea $(\geq 8)$, which showed no echocardiographic evidence of cardiovascular diseases; (2) an impaired relaxation group with a reduced Ea $(<8)$, a reduced E/A ratio $(<0.8)$, and a long DCT ( $>200 \mathrm{~ms})$; (3) a pseudonormal group with a reduced $\mathrm{Ea}(<8)$, an E/A ratio from 0.8 to 1.5 , and no shortening of DCT $(>160 \mathrm{~ms})$; and (4) a restrictive group with a reduced $\mathrm{Ea}(<8)$, an increased E/A ratio $(>2)$, and a shortened DCT $(<160 \mathrm{~ms})$.

\section{CMR Acquisition}

Supine patients were examined at rest using a 1.5-T scanner (Avanto; Siemens, Erlangen, Germany) with an 8-element phased-array body coil. Cine images were acquired at a rate of 25 phases per cardiac cycle by using segmented ECGtriggered, steady-state, free-precession cine imaging with echo sharing (true fast imaging with steady-state, free precession [True FISP] sequence), from the same 8-10 contiguous short-axis planes with an 8-mm section thickness and an inter-slice gap of $2 \mathrm{~mm}$, covering the entire LV from base to apex, as described previously [17].

\section{Evaluation of Cardiac Function by CMR}

CMR data were analyzed by two experienced observers who were blinded to the clinical backgrounds of the participants, using integral software (Argus; Siemens). The concepts of Echo-CK were applied to short-axis cine image datasets at the level of the mid-papillary muscle. We visually determined the end-diastolic and end-systolic phases and calculated the phase number during $30 \%$ of diastole by multiplying the total phase number during diastole by 0.3 and rounding up the values. We manually traced LV endocardial contours on only cine images of the end-diastolic phase, end-systolic phase, and the phase at $30 \%$ of diastole and then calculated the systolic fractional area change (systolic-index, percent) and fractional area change during the first $30 \%$ of diastole (diastolicindex, percent) (Figure 1).

\section{Reproducibility of CMR evaluation of cardiac function by fractional area change}

Intra-observer and inter-observer variability in the measurements of systolic and diastolic index was assessed in 20 randomly selected subjects. Two independent experienced observers who were blinded to each other's findings calculated the systolic and diastolic indexes. One of the two observers reanalyzed the results after an interval of approximately one week.

\section{Statistical analysis}

Data were statistically analyzed using GraphPad Prism (GraphPad software Inc., La Jolla, CA, USA) and are presented as means \pm standard deviation. The differences between the groups were assessed using one-way analysis of variance followed by the post-hoc test. Systolic and diastolic indexes were compared to conventional echocardiographic cardiac function measurements by using linear regression analysis with Pearson's correlation coefficient. Intra-observer and inter-observer variability in systolic and diastolic indexes were assessed by BlandAltman analysis. A p value of $<0.05$ was considered statistically significant.

\section{Results}

Patient characteristics (Table 1)

The 59 subjects were divided into four groups on the basis of echocardiographic findings: (1) the normal diastolic function group, 15 patients; (2) the impaired relaxation 


\section{Cine images ( 25 phases / cardiac cycle)}

End-diastole End-systole 30\%-diastole End-diastole
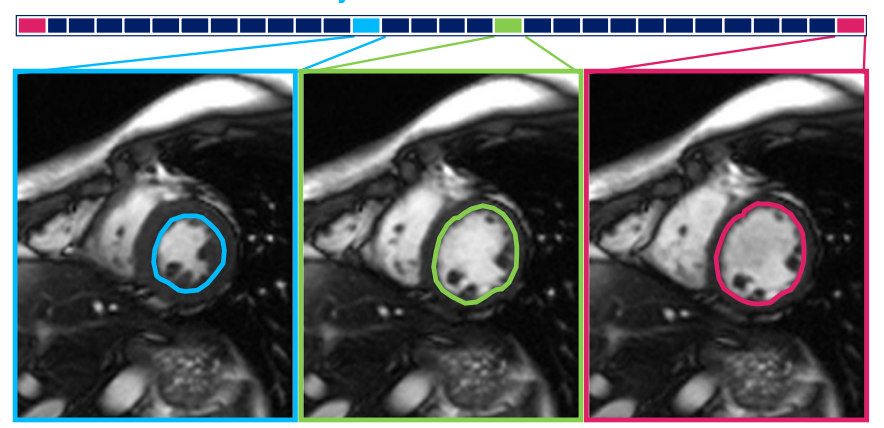

Tracking of LV

endomyocardial contours

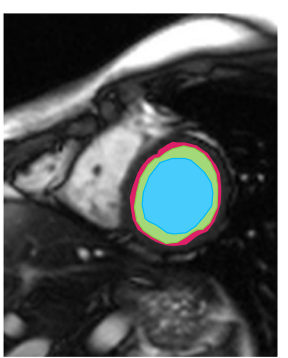

\section{Systolic index (\%)}

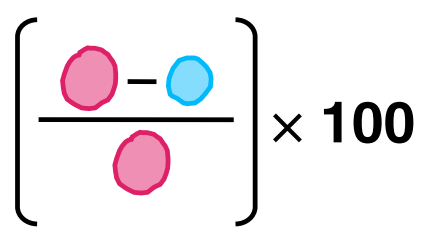

Diastolic index (\%)

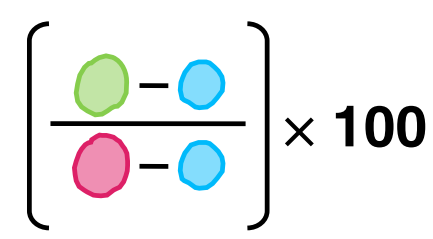

Figure 1 Evaluation of left ventricular systolic and diastolic function by fractional area change in cine CMR. End-diastolic and end-systolic phase are visually determined in datasets from short-axis cine images at mid papillary muscle, and phase number during $30 \%$ of diastole is calculated by multiplying total phase number during diastole by 0.3 and rounding up values. Left ventricular (LV) endocardial contours are then manually traced only on cine images of end-diastolic phase, end-systolic phase, and phase at $30 \%$ of diastole. The LV systolic fractional area change (systolic-index,\%) and fractional area change during the first 30\% of diastole (diastolic-index \%) are calculated.

Table 1 Clinical characteristics and echocardiographic variables in the four groups

\begin{tabular}{|c|c|c|c|c|}
\hline & Normal & Impaired relaxation & Pseudonormal & Restrictive \\
\hline Number & 15 & 28 & 11 & 5 \\
\hline Age (years) & $34.5 \pm 15.3$ & $71.1 \pm 9.9$ & $62.5 \pm 10.5$ & $63.0 \pm 10.1$ \\
\hline Male & 10 & 21 & 6 & 4 \\
\hline \multirow[t]{5}{*}{ Diagnosis } & & HHD 8 & IHD 3 & DHCM 1 \\
\hline & & IHD 9 & AS 1 & DCM 4 \\
\hline & & AS 1 & $\mathrm{HCM} 3$ & \\
\hline & & $\mathrm{HCM} 3$ & DCM 3 & \\
\hline & & & Amyloidosis 1 & \\
\hline \multicolumn{5}{|c|}{ Echocardiographic variables } \\
\hline LVEF (\%) & $65.9 \pm 6.2$ & $64.4 \pm 6.5$ & $56.9 \pm 15.0$ & $38.2 \pm 10.0$ \\
\hline LVDd (mm) & $46.6 \pm 4.4$ & $46.8 \pm 4.5$ & $55.2 \pm 7.5$ & $66.4 \pm 14.2$ \\
\hline LAD (mm) & $31.5 \pm 3.7$ & $38.1 \pm 4.7$ & $42.5 \pm 5.5$ & $47.2 \pm 7.5$ \\
\hline$E / A$ & $1.70 \pm 0.77$ & $0.69 \pm 0.11$ & $1.36 \pm 0.22$ & $2.24 \pm 0.54$ \\
\hline $\mathrm{DCT}$ (ms) & $205.6 \pm 34.9$ & $234.8 \pm 31.4$ & $190.2 \pm 23.5$ & $143.2 \pm 13.6$ \\
\hline Ea $(\mathrm{cm} / \mathrm{s})$ & $10.7 \pm 1.9$ & $6.3 \pm 1.4$ & $5.7 \pm 1.2$ & $4.9 \pm 1.6$ \\
\hline $\mathrm{E} / \mathrm{Ea}$ & $6.9 \pm 1.0$ & $9.5 \pm 2.6$ & $13.6 \pm 3.3$ & $20.4 \pm 8.2$ \\
\hline
\end{tabular}

Values are mean \pm standard deviation.

$H H D$ hypertensive heart disease, IHD ischemic heart disease, AS aortic valve stenosis, HCM hypertrophic cardiomyopathy, DCM dilated cardiomyopathy, $D H C M$ dilated-phase hypertrophic cardiomyopathy, LVEF left ventricular ejection fraction, LVDd left ventricular end-diastolic dimension, $L A D$ left atrial dimension; $\mathrm{E}$, peak early transmitral filling velocity; A, paek late transmitral filling velocity; DCT, deceleration time of peak E velocity; Ea, early diastolic mitral annular velocity. 
group, 28 patients; (3) the pseudonormal group, 11 patients; and (4) the restrictive group, 5 patients. The age was significantly lower in the normal diastolic function group than in the other three groups $(\mathrm{p}<0.0001)$. The LVEF was significantly lower in the restrictive group than in the other three groups $(\mathrm{p}<0.01)$. The LVDd were similar between the normal diastolic function group and impaired relaxation group, and $\mathrm{E} / \mathrm{Ea}$ increased significantly as diastolic dysfunction progressed from impaired relaxation to restrictive group $(p<0.05)$. The LAD was significantly smaller $(\mathrm{p}<0.001)$ and the Ea significantly higher, in the normal diastolic function group compared to the other three groups $(\mathrm{p}<0.001)$.

\section{LV systolic and diastolic index}

Figure 2 shows the comparison of systolic and diastolic indexes among the four groups. The LV systolic index was $54.0 \pm 7.2$ in the normal diastolic function group, $54.2 \pm 15.6$ in the impaired relaxation group, $39.4 \pm 15.8$ in the pseudonormal group, and $17.4 \pm 13.1$ in the restrictive group. The systolic index was significantly lower in the restrictive group than in the other three groups, and significantly lower in the pseudonormal group than in the normal diastolic function group and the impaired relaxation group. The LV diastolic index was $67.7 \pm 10.8$ in the normal diastolic function group, $32.4 \pm 7.5$ in the impaired relaxation group, $25.4 \pm 5.6$ in the pseudonormal group, and $9.5 \pm 1.5$ in the restrictive group. The diastolic index was significantly higher in the normal diastolic function group compared to the three groups of diastolic dysfunction and showed a significant reduction with the worsening of diastolic dysfunction.

Figure 3 shows the association between the LV systolic and diastolic indexes and conventional echocardiographic cardiac function measurements. The systolic index showed good positive correlation with EF $(\mathrm{r}=0.85, \mathrm{p}<0.0001)$, whereas the diastolic index showed moderate positive correlation with Ea $(\mathrm{r}=0.75, \mathrm{p}<0.0001)$ and negative correlation with $\mathrm{E} / \mathrm{Ea}(\mathrm{r}=0.58, \mathrm{p}<0.0001)$, but no correlation with $\mathrm{E} / \mathrm{A}(\mathrm{p}=0.14)$ and $\mathrm{DCT}(\mathrm{p}=0.35)$. The reduction of diastolic index was closely associated with the progression of LV diastolic dysfunction.

The intra-observer reproducibility of the systolic and diastolic indexes was $0.88 \pm 4.10 \%$ and $-0.12 \pm 5.62 \%$, respectively, while the corresponding inter-observer reproducibility was $3.40 \pm 5.90 \%$ and $3.03 \pm 11.1 \%$.

\section{Discussion}

This is the first study to show that the concepts of Echo-CK could be applied to MR cine imaging, and that LV diastolic and systolic function could be evaluated by the measurement of fractional area change. The degree of LV systolic and diastolic dysfunction evaluated by fractional area change in cine CMR correlated well with that determined by echocardiography. Evaluation of cardiac function by fractional area change is not new; however, it is considered to be useful because of the availability of a few simple techniques for the evaluation of diastolic function using CMR.
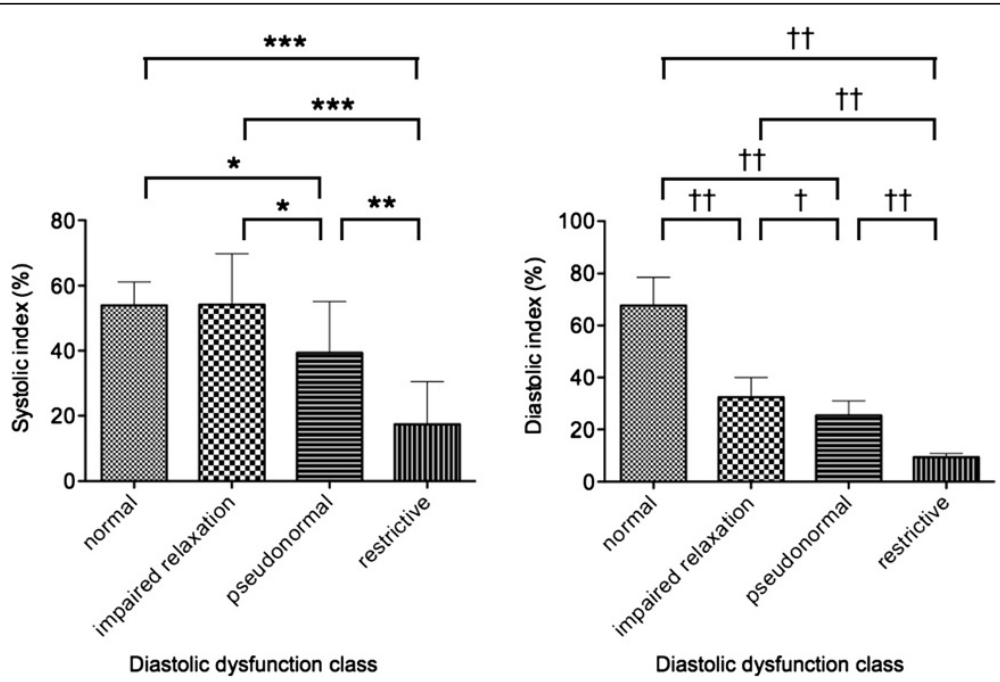

Figure 2 Comparison of the left ventricular (LV) systolic and diastolic indexes among the three groups. The systolic index was significantly lower in the restrictive group than in the other three groups, and significantly lower in the pseudonormal group than in the normal diastolic function group and the impaired relaxation group $\left({ }^{*} \mathrm{p}<0.05 ;{ }^{* *} \mathrm{p}<0.01 ;{ }^{* * *} \mathrm{p}<0.001\right)$. Whereas, the diastolic index was significantly higher in the normal diastolic function group compared to the three groups of diastolic dysfunction, and showed a significant reduction with the worsening of diastolic dysfunction $(+p<0.05 ; \dagger+p<0.001)$. 

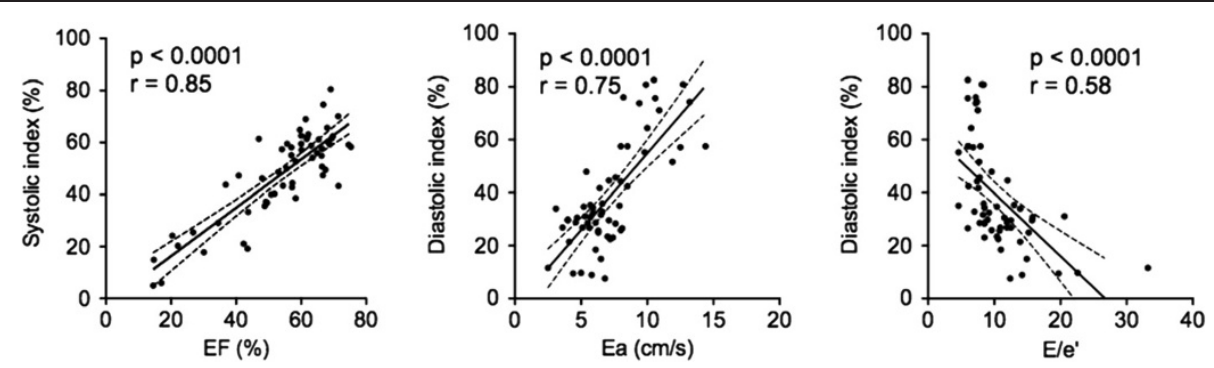

Figure 3 The association between the left ventricular (LV) systolic and diastolic indexes and conventional echocardiography cardiac function measurements. The LV systolic index well positively correlated with ejection fraction (EF), and the diastolic index moderately positively correlated with early diastolic mitral annular velocity (Ea) and negatively with the ratio of peak early filling velocity divided by Ea (E/Ea).

The original Echo-CK automatically traces the endocardial contours based on acoustic quantitation and is thus, difficult to apply to low-quality images due to a poor echo-window. In contrast, the quality of cine CMR is always excellent in individuals without arrhythmia who have no difficulty in breath-holding, and thus, we could easily apply the concepts of Echo-CK to cine image datasets obtained for all our study patients.

We defined the diastolic index as fractional area change during the first $30 \%$ of the diastole for the following reasons, as explained by the echocardiographic studies [11-15]. LV diastolic function consists of relaxation and filling, and the diastolic phase consists of an isovolumic relaxation phase and a filling phase in which blood flows from the LA to the LV. The isovolumic relaxation phase is a highly energy-demanding process and can be delayed by a slight deficit of myocardial energy. The fractional area change during the first $30 \%$ of diastole, mainly representing early regional LV relaxation, which occurs during isovolumic relaxation and early diastolic filling, is thus considered to be a more sensitive indicator of diastolic dysfunction. In this study, the systolic and diastolic indexes derived by CMR were $54.0 \pm 7.2$ and $67.7 \pm 10.8$ in patients with normal diastolic function, respectively. The diastolic index could differentiate between the normal and pseudonormalized pattern of LV inflow and classify the severity of diastolic dysfunction. The diastolic index correlated well with $\mathrm{Ea}$ and $\mathrm{E} / \mathrm{Ea}$, but not with E/A and DCT on echocardiography. We consider that the diastolic index reflects more directly the early diastolic myocardial motion than the transmitral flow determined by multiple parameters, such as filling pressure, LV elastic recoil, myocardial relaxation, and atrial and chamber compliance. These results agree with those of the echocardiographic studies [11-15]. Harada et al. concluded that the original Echo-CK can quantitatively evaluate the LV diastolic function by enabling the detection of regional LV delayed relaxation.
Evaluation of LV diastolic function by fractional area change in cine CMR has two advantages. First, it does not entail the expense of development of an acquisition sequence or post-processing software, and LV diastolic function can be easily evaluated by the existing equipment. Second, LV diastolic function can be retrospectively evaluated if prior cine image datasets are stored. In contrast, this method has inherent disadvantages, and four disadvantages similar to the original Echo-CK $[10,11]$. First, manual tracing of the LV endocardial contours in cine CMR images may limit the variability in the spatial and temporal resolution, which influences reproducibility to some extent. Therefore, we believe that the automatic tracing software needs to be developed to resolve this non-negligible problem. Second, the method simply evaluates the motion of an endocardial contour in a slice and ignores the influence of cardiac translation and/or rotation. However, the influence is considered to be relatively small because this method evaluates endocardial motion rather than wall thickening. Third, the method assesses regional, not global cardiac function. Fourth, clear images with minimum noise are needed. Naturally, a more detailed evaluation of cardiac function requires not only the measurement of fractional area change but echocardiography or CMR studies such as volumetric filling curves, phase-contrast imaging, tagging, and strain-encoded imaging.

The present study has the following four limitations. First, we retrospectively evaluated the data from a relatively small sample cohort at a single center. Second, a hemodynamic study was not performed. Third, the original Echo-CK method was not employed. Fourth, LV fractional area change was not evaluated in vertical or horizontal long-axis views. The changes in the vertical or horizontal long-axis views might not be similar to those in the short-axis view because the heart has a complex three-dimensional structure consisting of myocardial fibers oriented in different directions [18]. Evaluation 
of LV function by fractional area change in cine CMR should be prospectively tested in many more patients with several cardiac diseases at several centers.

\section{Conclusions}

The concepts of Echo-CK were applied to cine CMR, and the measurement of LV fractional area change on cine CMR can be employed as a screening tool to detect diastolic as well as systolic dysfunction.

\section{Consent}

Written informed consent could not be obtained from the subjects for the publication of this report and any accompanying images because the study was retrospectively designed and performed. However, the identity of the subjects are protected in this report.

\section{Abbreviations \\ CMR: Cardiovascular magnetic resonance; DCT: Deceleration time; EF: Ejection fraction; FAC: Fractional area change; LA: Left atrium; LV: Left ventricular; MR: Magnetic resonance.}

\section{Competing interests}

The authors declare that they have no competing interests.

\section{Authors' contributions}

SO; Analysis, and drafting the manuscript or revising it. TN; Analysis and interpretation of data. SU; Helping to draft the manuscript. SF; Contributions to conception and design. SS; Statistics. MW; Acquisition of data. TN; Acquisition of data. YS; Contributions to conception and design. All authors read and approved the final manuscript.

\section{Author details}

${ }^{1}$ First Department of Internal Medicine, Nara Medical University, Nara, Japan.

${ }^{2}$ Education Development Center, Nara Medical University, Nara, Japan.

${ }^{3}$ Department of Cardiology, Hirai Hospital, Nara, Japan.

Received: 2 July 2013 Accepted: 17 September 2013

Published: 26 September 2013

\section{References}

1. Oki T, Tabata T, Yamada H, Wakatsuki T, Shinohara H, Nishikado A, luchi A, Fukuda N, Ito S. Clinical application of pulsed Doppler tissue imaging for assessing abnormal left ventricular relaxation. Am J Cardiol. 1997; 79:921-28.

2. Zile MR, Baicu CF, Gaasch WH. Diastolic heart failure-abnormalities in active relaxation and passive stiffness of the left ventricle. $N$ Engl J Med. 2004; 350:1953-59.

3. Redfield MM, Jacobsen SJ, Burnett JC Jr, Mahoney DW, Bailey KR, Rodeheffer RJ. Burden of systolic and diastolic ventricular dysfunction in the community: appreciating the scope of the heart failure epidemic. JAMA. 2003; 289:194-202.

4. Ogunyankin KO. Assessment of left ventricular diastolic function: the power, possibilities, and pitfalls of echocardiographic imaging techniques. Can J Cardiol. 2011; 27:311-18.

5. Kawaji K, Codella NC, Prince MR, Chu CW, Shakoor A, LaBounty TM, Min JK, Swaminathan RV, Devereux RB, Wang Y, Weinsaft JW. Automated segmentation of routine clinical cardiac magnetic resonance imaging for assessment of left ventricular diastolic dysfunction. Circ Cardiovasc Imaging. 2009; 2:476-84.

6. Rathi VK, Doyle M, Yamrozik J, Williams RB, Caruppannan K, Truman C, Vido D, Biederman RW. Routine evaluation of left ventricular diastolic function by cardiovascular magnetic resonance: a practical approach. J Cardiovasc Magn Reson. 2008; 10:36

7. Fernandes VR, Edvardsen T, Rosen BD, Carvalho B, Campos O, Cordeiro MA, Kronmal R, Bluemke DA, Lima JA. The influence of left ventricular size and global function on regional myocardial contraction and relaxation in an adult population free of cardiovascular disease: a tagged CMR study of the MESA cohort. J Cardiovasc Magn Reson. 2007; 9:921-30.

8. Neizel M, Korosoglou G, Lossnitzer D, Kühl H, Hoffmann R, Ocklenburg C, Giannitsis E, Osman NF, Katus HA, Steen H. Impact of systolic and diastolic deformation indexes assessed by strain-encoded imaging to predict persistent severe myocardial dysfunction in patients after acute myocardial infarction at follow-up. J Am Coll Cardiol. 2010; 56:1056-62.

9. Natsume T, Amano T, Takehara Y, Ichihara T, Takeda K, Sakuma H. Quantitative assessment of regional systolic and diastolic functions and temporal heterogeneity of myocardial contraction in patients with myocardial infarction using cine magnetic resonance imaging and Fourier fitting. Magn Reson Imaging. 2009; 27:1440-46.

10. Lang RM, Vignon P, Weinert L, Bednarz J, Korcarz C, Sandelski J, Koch R, Prater D, Mor-Avi V. Echocardiographic quantification of regional left ventricular wall motion with color kinesis. Circulation. 1996; 93:1877-85.

11. Vignon P, Mor-Avi V, Weinert L, Koch R, Spencer KT, Lang RM. Quantitative evaluation of global and regional left ventricular diastolic function with color kinesis. Circulation. 1998; 97:1053-61.

12. Miwa K, Ishii K, Makita T, Okuda N. Effects of postischemic regional left ventricular diastolic wall motion abnormalities or delayed relaxation following coronary vasospasm on global diastolic function. Circ J. 2005; 69:439-45.

13. Harada M, Hayashi K, Takarada Y, Hirai H. Evaluation of left ventricular diastolic function using color kinesis. J Med Ultrasonics. 2007; 34:29-35.

14. Harada M, Hara F, Hayashi K, Takarada Y. Assessment of left ventricular diastolic function using color kinesis: differentiation between normal and pseudonormalized patterns. J Med Ultrasonics. 2009; 36:69-75.

15. Takeda Y, Sakata Y, Mano T, Nishio M, Ohtani T, Hori M, Masuyama T, Yamamoto K. Noninvasive assessment of diastolic function in subjects with preserved left ventricular ejection fraction: usefulness of color kinetic imaging. J Card Fail. 2008; 14:569-76.

16. Nagueh SF, Appleton CP, Gillebert TC, Marino PN, Oh JK, Smiseth OA, Waggoner AD, Flachskampf FA, Pellikka PA, Evangelisa A. Recommendations for the evaluation of left ventricular diastolic function by echocardiography. Eur J Echocardiogr. 2009; 10:165-93.

17. Okayama S, Uemura S, Soeda T, Onoue K, Somekawa S, Ishigami K, Watanabe M, Nakajima T, Fujimoto S, Saito Y. Clinical significance of papillary muscle late enhancement detected via cardiac magnetic resonance imaging in patients with single old myocardial infarction. Int J Cardiol. 2011; 146:73-9.

18. Buckberg GD, Mahajan A, Jung B, Markl M, Hennig J, Ballester-Rodes M. MRI myocardial motion and fiber tracking: a confirmation of knowledge from different imaging modalities. Eur I Cardiothorac Surg. 2006; 29(Suppl 1):165-77.

\section{doi:10.1186/1532-429X-15-87}

Cite this article as: Okayama et al:: Evaluation of left ventricular diastolic function by fractional area change using cine cardiovascular magnetic resonance: a feasibility study. Journal of Cardiovascular Magnetic Resonance 2013 15:87.

\section{Submit your next manuscript to BioMed Central and take full advantage of:}

- Convenient online submission

- Thorough peer review

- No space constraints or color figure charges

- Immediate publication on acceptance

- Inclusion in PubMed, CAS, Scopus and Google Scholar

- Research which is freely available for redistribution 